\title{
Caracterização da Região 3'-Terminal do Genoma de um Isolado Brasileiro do Southern bean mosaic virus*
}

\author{
Luciana M. Espinha ${ }^{1 * *}$, José O. Gaspar ${ }^{1 * * *}$, Andreia E. Moreira ${ }^{1}$, Ana Cecília B. Pereira ${ }^{1 * * *}$, \\ Priscila Belintani $^{1 * * *}$ \& Luis E. A. Camargo ${ }^{2}$ \\ ${ }^{1}$ Departamento de Zoologia e Botânica, IBILCE-UNESP, CEP 15054-000, São José do Rio Preto, SP, \\ e-mail: gaspar@ibilce.unesp.br; ${ }^{2}$ Departamento de Entomologia, Fitopatologia e Zoologia Agrícola, \\ ESALQ-USP, Cx. Postal 9, CEP 13418-900, Piracicaba, SP
}

(Aceito para publicação em 13/07/2005)

Autor para correspondência: José Osmar Gaspar

ESPINHA, L.M., GASPAR, J.O., MOREIRA, A.E., PEREIRA, A.C.B., BELINTANI, P. \& CAMARGO, L.E.A. Caracterização da região 3'-terminal do genoma de um isolado brasileiro do Southern bean mosaic virus. Fitopatologia Brasileira 30:546-549. 2005.

\section{RESUMO}

O presente trabalho caracteriza a região 3'-terminal do genoma de um isolado do Southern bean mosaic virus encontrado no Estado de São Paulo (SBMV-SP). O RNA foi extraído de partículas virais purificadas e submetido a RTPCR usando oligonucleotídeos desenhados para amplificar $972 \mathrm{nt}$ da região 3'-terminal do RNA viral. Foi obtido fragmento de tamanho esperado que inclui o gene da proteína capsidial e a região 3'-terminal não codificadora. O gene da proteína capsidial (ORF4) contém 801 nucleotídeos, incluindo-se o códon de terminação UGA, com seqüência deduzida de 266 aminoácidos e massa molecular estimada de 28.800 Da. Sessenta e um aminoácidos terminais da ORF2 estão sobrepostos na ORF4. O "sinal de localização nuclear", encontrado dentro do "Domínio R" na região 5'-terminal da ORF4 de alguns sobemovírus, não foi identificado no SBMV-SP. Esse dado pode explicar a ausência de partículas virais do SBMV-SP no núcleo celular. A seqüência do SBMV-SP apresentou identidade de nucleotídeos e aminoácidos de, respectivamente, 91\% e 93\% com o isolado "Arkansas" (SBMV-ARK) descrito nos EUA. Os resultados obtidos indicam que o SBMV-SP e o SBMV-ARK são isolados muito proximamente relacionados.

Palavras-chave adicionais: feijoeiro comum, Sobemovirus, SBMV, seqüenciamento.

\begin{abstract}
Characterization of the 3'-terminal genome region of a Brazilian isolate of Southern bean mosaic virus

We report the characterization of the 3'-terminal genome region of an isolate of Southern bean mosaic virus found in the State of São Paulo, Brazil (SBMV-SP). The RNA was extracted from purified virus particles and subjected to RT-PCR using oligonucleotides designed to amplify $972 \mathrm{nt}$ of the 3'-terminal region of the viral genome. The coat protein gene (ORF4) contains $801 \mathrm{nt}$, including the stop codon UGA, coding for 266 amino acids with a predicted molecular weight of 28,800 Da. The 3'-terminus of ORF2 overlaps the 5'-terminus of ORF4 in 61 amino acids. The nuclear localization signal sequence, found in the "R domain" at the 5'-terminal of the ORF4 in some sobemoviruses, was not identified in SBMV-SP. This could explain the absence of SBMV-SP particles inside the cell nucleus. The SBMV-SP sequence showed identity of nucleotides and amino acids of, respectively, $91 \%$ and $93 \%$ with the "Arkansas" isolate (SBMV-ARK) described in the USA. The results obtained in the present work indicate that SBMV-SP and SBMV-ARK are closely related isolates of the same virus.
\end{abstract}

Additional keywords: commom bean, Sobemovirus, SBMV, sequencing.

O isolado americano do Southern bean mosaic virus (SBMV), gênero Sobemovirus, já se encontra bem caracterizado, havendo relatos sobre a estrutura do vírion (Hull, 1977), propriedades da capa proteica (Sheperd, 1971), do genoma (Weber \& Sehgal, 1982), seqüenciamento e tradução in vitro de cada uma das ORFs (Othman \& Hull, 1995) e interação com a planta hospedeira através de

\footnotetext{
* Parte da Tese de Doutorado da primeira autora. Universidade Estadual Paulista (2003). Apoio Financeiro: FAPESP, CAPES. Seqüência depositada no GenBank com o número de acesso AY340586;

** Bolsista da CAPES;

**** Bolsista do CNPq
}

microscopia eletrônica de transmissão (Weintraub \& Ragetli, 1970).

O genoma dos sobemovírus possui quatro ORFs que se sobrepõem (Van Regenmortel et al., 2000), com exceção para o SBMV que possui somente três ORFs não sobrepostas (Othman \& Hull, 1995). Ao SBMV falta a pequena ORF3 que é, entretanto, encontrada no isolado "Arkansas" (SBMVARK; Lee \& Anderson, 1998). Foi proposto (Lee \& Anderson, 1998) que as diferenças na organização do genoma entre o SBMV e o SBMV-ARK resultariam de mutações ou erros no seqüenciamento de nucleotídeos do SBMV. 
No Brasil, o SBMV foi detectado pela primeira vez em feijoeiros (Phaseolus vulgaris L.) na região do Distrito Federal (SBMV-DF; Cupertino et al., 1982) e, posteriormente, mas ainda nos anos 80, no Estado de São Paulo (SBMV-SP; A. S. Costa, dados não publicados). Mais recentemente, o vírus foi também encontrado no Estado do Paraná (SBMV-PR, Gasparin et al., 2005). Algumas propriedades moleculares foram determinadas para o isolado SBMV-SP: (a) as partículas virais apresentam diâmetro de $28-30 \mathrm{~nm}$ e a proteína capsidial possui massa molecular de $30 \mathrm{kDa}$; (b) das partículas virais foram extraídos RNAs de vários tamanhos, sendo o de 4,2 Kb o RNA genômico e o de 1,0 $\mathrm{Kb}$ supostamente um RNA subgenômico que codifica a proteína capsidial (Moreira \& Gaspar, 2002).

A região 5'-terminal do SBMV-SP foi caracterizada (Espinha et al., 2004), evidenciando-se uma região não codificadora, com 92 nt, precedendo o códon de iniciação da ORF1. Nesta região está contido um segmento parcialmente complementar ao RNA ribossomal. A ORF1 codifica uma proteína de 147 aminoácidos com massa molecular estimada em 17.080 Da. Foi também evidenciado que a extremidade 3' da ORF1 sobrepõe a ORF2.

No presente trabalho descreve-se a caracterização da região 3'-terminal do genoma do isolado do SBMV encontrado no Estado de São Paulo (SBMV-SP). Este isolado foi gentilmente cedido pelo Dr. Álvaro Santos Costa (Instituto Agronômico de Campinas), inoculado em feijoeiro comum cv. Rosinha e mantido em casa de vegetação com temperatura controlada ao redor de $28^{\circ} \mathrm{C}$.

$\mathrm{O}$ vírus foi purificado seguindo-se o protocolo descrito por Moreira \& Gaspar (2002). Para extração do RNA, uma alíquota do vírus purificado foi tratada com SDS $2,5 \%$ e $\mathrm{NaCl} 0,1 \mathrm{M}$ com aquecimento por 3 min à $55^{\circ} \mathrm{C}$. Em seguida foi acrescentado igual volume de fenol (equilibrado com tampão citrato de sódio $0,1 \mathrm{M}, \mathrm{pH} 4,3$ ), agitada manualmente por $30 \mathrm{~s} \mathrm{e}$, então, centrifugada para a separação das fases. A fase aquosa foi novamente extraída com fenol e, após centrifugação, o ácido nucleico foi precipitado da fase aquosa pela adição de $1 / 10$ do volume de acetato de sódio $3 \mathrm{M}, \mathrm{pH} 5,2$, e três volumes de etanol por $1 \mathrm{~h}$ à $-70{ }^{\circ} \mathrm{C}$. Em seguida, a mistura foi submetida à centrifugação a $12.000 \mathrm{~g}$ por $15 \mathrm{~min}$ e o "pellet" foi secado à $37^{\circ} \mathrm{C}$ por 10 min e solubilizado em água tratada com DEPC.

Para a produção do DNA complementar (cDNA) ao RNA viral, foi utilizado um volume de reação de $20 \mu \mathrm{l}$ consistindo de $50 \mathrm{mM}$ Tris- $\mathrm{HCl}$ (pH 8,3), $75 \mathrm{mM} \mathrm{KCl}, 3 \mathrm{mM}$ $\mathrm{MgCl}_{2}, 10 \mathrm{mM}$ DTT, $0,1 \mathrm{mM}$ de cada dNTP, $2 \mu \mathrm{d}$ do oligonucleotídeo anti-senso (descrito abaixo) na concentração de $10 \mathrm{pmol} / \mu \mathrm{l}$, duas unidades de inibidor de RNAse, $1-3 \mu \mathrm{g}$ de RNA viral desnaturado à $95^{\circ} \mathrm{C}$ por 5 min e 40 unidades de transcriptase reversa "Superscript II" (Invitrogen, Carlsbad, CA, EUA). A mistura de reação permaneceu por $2 \mathrm{~h}$ à $42^{\circ} \mathrm{C}$.

Para amplificar o gene da proteína capsidial foram utilizados oligonucleotídeos que cobrem a seqüência dos nucleotídeos 3164 a 4136, compreendendo região de 972 pares de bases, de acordo com a seqüência do SBMV-ARK
(Lee \& Anderson, 1998). A seqüência do par de oligonucleotídeos é: Senso, 5'-CATCGCCCCACAAAACTAAT-3' (localizado $42 \mathrm{nt}$ anteriores ao códon de iniciação da ORF4) e Anti-senso, 5'-ACTTTTGGATTACGCTCCATC-3' (localizado $108 \mathrm{nt}$ posteriores ao códon de terminação UGA da ORF4). O volume da reação da PCR foi de $25 \mu \mathrm{l}$ consistindo de tampão (Tris- $\mathrm{HCl} 20 \mathrm{mM} \mathrm{pH} \mathrm{8,4,} \mathrm{KCl} 50 \mathrm{mM}$ ), $5 \mathrm{mM}$ de $\mathrm{MgCl}_{2}, 0,2 \mathrm{mM}$ de cada dNTP, $2 \mu 1$ de cada oligonucleotídeo (senso e anti-senso) na concentração de $10 \mathrm{pmol} / \mu \mathrm{l} ; 1 \mu \mathrm{l}$ da mistura da reação de cDNA e duas unidades de Taq DNA polimerase (Invitrogen). O programa da PCR, realizado em um termociclador "MiniCycler" (MJ Research, San Francisco, CA, EUA) envolveu uma desnaturação inicial a $94{ }^{\circ} \mathrm{C}$ por 4 min e 30 ciclos com 1 min de desnaturação a $94^{\circ} \mathrm{C}, 1 \mathrm{~min}$ de anelamento a $45^{\circ} \mathrm{C}$ e $1 \mathrm{~min}$ de extensão a $72{ }^{\circ} \mathrm{C}$. Após os 30 ciclos foi feita a extensão final por 10 min a $72{ }^{\circ} \mathrm{C}$. Os fragmentos de DNA amplificados foram separados em gel de agarose $1 \%$, corado com brometo de etídeo e visualizados sob luz ultravioleta.

O produto de amplificação foi clonado utilizando-se o "SureClone ${ }^{\mathrm{TM}}$ Ligation Kit" (Amersham Biosciences, San Francisco, CA, EUA) e a extração do plasmídeo recombinante realizada com o "Concert Rapid Plasmid Miniprep System Kit" (Invitrogen) de acordo com instruções dos fabricantes. Após a extração do DNA plasmidial das células selecionadas, foram feitas digestões com as enzimas de restrição EcoRI e BamHI para comprovar a formação do recombinante. Os DNAs plasmidiais purificados foram seqüenciados pela técnica de terminação em cadeia utilizando-se o seqüenciador automático ABI Prism 377 (Applied Biosystems, Norwalk, CT, EUA) e o "Big Dye Terminator Cycle Sequencing Ready Reaction Kit" (Applied Biosystems), seguindo-se as recomendações do fabricante. $\mathrm{O}$ seqüenciamento de cinco clones foi realizado nos dois sentidos de leitura. Análises comparativas com seqüências existentes no "GenBank" foram feitas através do algoritmo BLAST (http://www.ncbi.nlm.nih.gov).

$\mathrm{O}$ alinhamento das seqüências foi efetuado pelo programa CLUSTAL W (http://www.ch.embnet.org/ software/ClustalW.html) com o objetivo de obter relações filogenéticas pelo método "neighbor-joining", como implementado no programa PAUP v.4.0b10 (Swofford, 1997). A análise de "bootstrap" consistiu de 500 réplicas e a árvore foi construída usando o programa Tree View 1.6.1. (http://taxonomy.zoology.gla.ac.uk/rod/treeview.html).

A ORF 4 (gene da proteína capsidial) do SBMV-SP contém 801 nucleotídeos, incluindo-se o códon de terminação UGA, com seqüência deduzida de 266 aminoácidos e massa molecular estimada de $28.800 \mathrm{Da}$. A seqüência do gene codificador da proteína capsidial do SBMV-SP (Figura 1), quando comparada com seqüências depositadas no GenBank (Tabela 1), mostrou que este isolado é mais proximamente relacionado ao isolado "Arkansas" descrito nos EUA (SBMV-ARK; Lee \& Anderson, 1998). Existe entre esses isolados $91 \%$ de identidade na seqüência de nucleotídeos e $93 \%$ de identidade na seqüência de aminoácidos. Em ordem 
TABELA 1 - Porcentagens de identidade de nucleotídeos (nt) e aminoácidos (aa) entre o isolado de Southern bean mosaic virus (SBMV-SP) e outros sobemovírus. Os números de acesso ao GenBank para os vírus citados são aqueles apresentados na Figura 2

\begin{tabular}{lccc}
\hline \hline \multirow{2}{*}{ Isolado comparado } & $\begin{array}{c}\text { Região 3' não } \\
\text { codificadora }\end{array}$ & \multicolumn{2}{c}{ ORF4 } \\
& 97 & 91 & 93 \\
\hline SBMV-ARK & 94 & 83 & 84 \\
SBMV & 29 & 76 & 75 \\
SeMV & 22 & 66 & 66 \\
SCPMV &
\end{tabular}

*SBMV-ARK - Southern bean mosaic virus Arkansas; SBMV - Southern bean mosaic virus; SeMV - Sesbania mosaic virus; SCPMV - Southern cowpea mosaic virus

decrescente de identidade, dentro da mesma família, estão o SBMV (Othman \& Hull, 1995), o Sesbania mosaic virus (SeMV), o Southern cowpea mosaic virus (SCPMV) e o Lucerne transient streak virus (LTSV). Seqüências de outros sobemovírus analisados não mostraram identidade significativa com o SBMV-SP. A análise filogenética (Figura 2A) realizada com base nas seqüências de aminoácidos correspondentes às proteínas capsidiais de alguns sobemovírus agrupou o SBMV-SP e o SBMV-ARK no mesmo ramo em $69 \%$ das réplicas, indicando maior proximidade entre eles. O agrupamento do SBMV-SP e SBMV-ARK apresentou-se fortemente relacionado com o SBMV $(100 \%)$ e o SeMV (91\%).

Os 61 aminoácidos terminais da ORF2 sobrepõem a ORF 4 e apresentam $92 \%$ de identidade com o SBMV-ARK (Figura 1). Essa sobreposição, assim como aquela existente entre as ORF1 e a ORF2 do SBMV-SP (Espinha et al., 2004), corroboram a proposta de Lee \& Anderson (1998) de que deve ter ocorrido erro no seqüenciamento do SBMV (Othaman \& Hull, 1995), já que este isolado apresenta somente três ORFs que não se sobrepõem, diferenciando-se dos demais sobemovírus que possuem quatro ORFs sobrepostas.

A região 3' não codificadora do SBMV-SP apresenta 129 nucleotídeos (Figura 1) e, ao ser comparada com outros isolados e espécies de sobemovírus, apresentou 97\% de identidade com o isolado Arkansas, 94\% com o SBMV, $29 \%$ com o SeMV e $22 \%$ com o SCPMV (Tabela 1 ).

O domínio R, localizado próximo ao terminal 5' da ORF 4, é uma região da cadeia polipeptídica rica em arginina, lisina, prolina e glutamina, voltada para o interior da partícula, onde interage com o RNA (Abad-Zapatero et al., 1980). Nessa região, os genomas do SBMV e do SCPMV apresentam um sinal de localização nuclear (Dingwall \& Laskey, 1991), o qual poderia estar relacionado com a presença de partículas desses dois vírus no núcleo celular, um fato já demostrado por Weintraub \& Ragetli (1970). O alinhamento (Figura $2 \mathrm{~B}$ ) das regiões do domínio $\mathrm{R}$ do SBMV-SP, SBMV-ARK, SBMV e SCPMV mostrou haver
001 AUGGCGAAGAGGCUGACAAAACAACAGUUAGCCAAGGCUA $\begin{array}{llllllllllllllllll}M & A & K & R & L & T & K & Q & Q & L & A & K & A & I\end{array}$

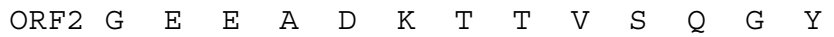
041 UAGCGAACACUCUGGAAGCCCCGGCCACUCAAUCGAGGAG $\begin{array}{lllllllllllll}A & N & T & L & E & A & P & A & T & Q & S & R & R\end{array}$ $\begin{array}{lllllllllllllllllllllllll}S & E & H & S & G & S & P & G & H & S & I & E & E\end{array}$ 081 GCCCAGGAACCGGAGACGGCGCCGUUCUGCUGCAAGGCAG $\begin{array}{lllllllllllll}P & R & N & R & R & R & R & R & S & A & A & R & Q\end{array}$

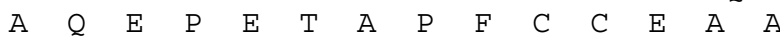
121CCUCAGUCUAUCCAGGCUGGGGCAUCCAUGGCCCCUAUUG $\begin{array}{llllllllllllll}P & Q & S & I & Q & A & G & A & S & M & A & P & I & A\end{array}$

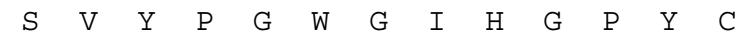
161CUCAGGGGGCUAUGGUUCGCUUACGUGAACCAUCGCUUAG $\begin{array}{llllllllllllll}Q & G & A & M & V & R & L & R & \bar{E} & P & S & L & R\end{array}$ $S \quad G \quad G \quad Y \quad G \quad S \quad L \quad T \quad$ *

201 AACGGCUGGAGGAGUGACUGUCCUGACGCACUCUGAGCUC $\begin{array}{lllllllllllll}T & A & G & G & V & T & V & L & T & H & S & E & L\end{array}$ 241 UCAACAGAGCUUGCUGUGACGAAUGCGAUAGUUGUCUCUU $\begin{array}{llllllllllllll}S & T & E & L & A & V & T & N & A & I & V & V & S & S\end{array}$ 281 CUGAGCUUGUCAUGCCCUUCACAAUGGGCACUUGGCUUCG $\begin{array}{lllllllllllll}E & \mathrm{~L} & \mathrm{~V} & \mathrm{M} & \mathrm{P} & \mathrm{F} & \mathrm{T} & \mathrm{M} & \mathrm{G} & \mathrm{T} & \mathrm{W} & \mathrm{L} & \mathrm{R}\end{array}$ 321 AGGCGUGGCGGCCAAUUGGUCGAAGUACAGUCUGUUUUCA $\begin{array}{lllllllllllll}G & V & A & A & N & W & S & K & Y & S & L & F & S\end{array}$ 361 GUGAGGUAUACUUACCUCCCCUCUUGUCCUUCAACGACAU $\begin{array}{llllllllllllll}V & R & Y & T & Y & L & P & S & C & P & S & T & T & S\end{array}$ 401 CUGGGUCCAUUCAUAUGGGUUUCCAAUAUGAUAUGGCUGA $\begin{array}{lllllllllllll}G & S & I & H & M & G & F & Q & Y & D & M & A & D\end{array}$ 441 CACUCUUCCCGUAUCCGUUAACCAGUUAUCCAACCUUAGA $\begin{array}{llllllllllllll}T & L & P & V & S & V & N & Q & L & S & N & L & R\end{array}$ 481 GGCUAUGUGUCAGGGCAGGUCUGGUCUGGAUCCUCUGGAU $\begin{array}{llllllllllllll}G & Y & V & S & G & Q & V & W & S & G & S & S & G & L\end{array}$ 521 UGUGCUAUAUAAAUGGCACGAGGUGUUCUGACACCGCCAA $\begin{array}{lllllllllllll}C & Y & I & N & G & T & R & C & S & D & T & A & N\end{array}$ 561CGCUAUCACGACCACUUUGGACGUUGCAAAGCUUGGUAAG $\begin{array}{lllllllllllll}A & I & T & T & T & L & D & V & A & K & L & G & K\end{array}$ 601 AAGUGGUAUCCUUUCAAGACCAGUACGGACUUCACUGCCG $\begin{array}{llllllllllllll}K & W & Y & P & F & K & T & S & T & D & F & T & A & A\end{array}$ 641 CUGUUGGCGUAAUAGUCAACAUUGCUACUCCCCUGGUCCC $\begin{array}{lllllllllllll}V & G & V & I & V & N & I & A & T & P & L & V & P\end{array}$ 681GGCUAGGCUAGUGAUAGCCAUGCUGGAUGGGUCGAGUUCU $\begin{array}{lllllllllllll}A & R & L & V & I & A & M & L & D & G & S & S & S\end{array}$ 721ACGGCUGUGAGUACUGGACGCCUAUACGUGUCGUACACUG $\begin{array}{llllllllllllll}T & A & V & S & T & G & R & L & Y & V & S & Y & T & V\end{array}$ 761 UACAGCUAAUUGAGCCGACUGCCUUGGCCUUAAACAACUG $Q \begin{array}{llllllllllll}\mathrm{L} & \mathrm{I} & \mathrm{E} & \mathrm{P} & \mathrm{T} & \mathrm{A} & \mathrm{L} & \mathrm{A} & \mathrm{L} & \mathrm{N} & \mathrm{N} & \text { * }\end{array}$ 801 AGGAGUUGUAUAAUAAUACCUGCACCCUUCUCUUUGGCAG $841 \bar{G}$ GAGGGUGUUUCGUUUUCACAAUGCCACGCGCUUGAGGGA $881 \mathrm{GAAUGCACGUUAAUCAUCCCUCCGCUAGUGAUGGAGCGUA}$ 921AUCCAAAAGU

FIG. 1 - Seqüência de nucleotídeos e aminoácidos deduzidos da região 3' terminal do Southern bean mosaic virus (SBMV-SP). Os últimos 129 nucleotídeos representam a região não codificadora. Os códons de iniciação (AUG) e de terminação (UGA) da ORF 4 estão sublinhados. Os últimos 61 aminoácidos da ORF 2 (sobreposta na ORF 4) estão mostrados na linha inferior aos aminoácidos da ORF 4. O códon de terminação da ORF 2 está duplamente sublinhado.

diferença não só no número (cinco a mais no SBMV-SP e SBMV-ARK) como na seqüência de aminoácidos. SBMV e SCPMV possuem o sinal de localização nuclear do tipo bipartido (consenso: $(\mathrm{K} / \mathrm{R})_{2} \mathrm{X}_{10-12}(\mathrm{~K} / \mathrm{R})_{3}$ ), ausente no SBMV- 
A

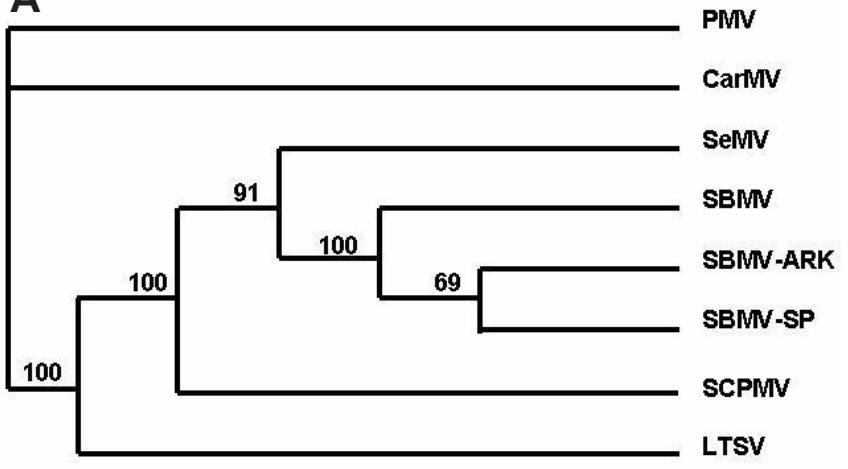

B

SBMV-SP

SBMV-ARK

SBMV

SCPMV
TKQQLAKAIANTLEAPATQSRRPRNRRR TKQQLTKAIANTLEAPATQSRRPRNRRR TKKQLAQAIQNTLPNPP _ RRKRRA TKKQLAQAIQNTLPNPP_RRKRRA_ $* *$ $* * * * *$

FIG. 2 - (A) Análise filogenética com base na seqüência completa de aminoácidos da proteína capsidial do Southern bean mosaic virus (SBMV-SP) e outros sobemovírus. Os números de acesso ao GenBank para os vírus citados acima são: Lucerne transiente streak virus (LTSV) (NC001696), Sesbania mosaic virus (SeMV) (NC002568), SBMV (L34672), SBMV-ARK (NC004060), SBMV-SP (AY340586), Southern caupi mosaic virus (SCPMV) (NC001625), Panicum mosaic virus (PMV) (NC002598) e Carnation mottle virus (CarMV) (NC001265). (B) Alinhamento do "sinal de localização nuclear" na seqüência de nucleotídeos de alguns sobemovírus. Os asteriscos indicam resíduos de aminoácidos que caracterizam o sinal de localização nuclear do tipo bipartido.

SP e SBMV-ARK. Estudos preliminares ao microscópio eletrônico e de fracionamento celular sugeriram que o SBMV-SP está ausente do núcleo de células infettadas, mas não há estudos similares com o SBMV-ARK. A ausência do SBMV-SP no núcleo celular é ainda mais instigante levandose em conta o fato de que Muñoz \& Kitajima (1990) citaram a presença, no núcleo celular, de partículas do isolado do SBMV encontrado no Distrito Federal (SBMV-DF). Isso abre a possibilidade de que ocorram diferentes isolados do SBMV no Brasil e o seqüenciamento do gene da proteína capsidial do SBMV-DF poderá elucidar essas questões.

\section{REFERÊNCIAS BIBLIOGRÁFICAS}

ABAD-ZAPATERO, C., ABDEL-MEGUID, S.S., JOHNSON, J.E.,

LESLIE, A.G.W., RAYMENT, I., ROSSMAN, M.G., SUCK, D. \& TSUKUHARA, T. Structure of Southern bean mosaic virus at 2.8 angstrom resolution. Nature 286:33-39. 1980.

CUPERTINO, F.P., LIN, M.T., KITAJIMA, E.W. \& COSTA, C.L. Occurrence of southern bean mosaic virus in central Brazil. Plant Disease 66:742-743. 1982.

DINGWALL, C. \& LASKEY, R.A. Nuclear targeting sequence A consensus. Trends in Biochemistry Science 16:478-481. 1991. ESPINHA, L.M., GASPAR, J.O., WARD, R.J., RULLER, R. \& CARMARGO, L.E.A. Caracterização da região 5'-terminal de um isolado brasileiro do Southern bean mosaic virus. Fitopatologia Brasileira 29:328-331. 2004.

GASPARIN, M.D.G., BIANCHINI, A., DE SOUTO, E.R., GASPAR, J.O., ALMEIDA, A.M.R. \& KITAJIMA, E.W. Detecção do Southern bean mosaic virus no Paraná, e Separação do Bean rugose mosaic virus em Feijoeiro. Fitopatologia Brasileira 30:7578. 2005.

HULL, R. The grouping of small spherical plant viruses with single RNA components. Journal of General Virology 36: 289. 1977.

LEE, L. \& ANDERSON, J. Nucleotide sequence of a resistance breaking mutant of Southern bean mosaic virus. Archives of Virology 143:2189-2201. 1998.

MOREIRA, A.E. \& GASPAR, J.O. Propriedades moleculares de um isolado brasileiro do Southern bean mosaic virus. Fitopatologia Brasileira 27:292-297. 2002.

MUÑOZ, J.O. \& KITAJIMA, E.W. Estudo comparativo da citopatologia induzida por alguns vírus do feijoeiro (Phaseolus vulgaris) em infecções simples. Fitopatologia Brasileira 15:276284. 1990.

OTHMAN, Y. \& HULL, R. Nucleotide sequence of the bean strain of Southern bean mosaic virus. Virology 206:287-297. 1995.

SHEPHERD, R.J. Southern bean mosaic virus. In: Description of Plant Viruses. Commonwealth Mycological Institute/Association of Applied Biologists Kew, Surrey, England, n 57. 1971.

SWOFFORD, D. PAUP: Phylogenetic analysis using parsimony. Version 4.0b10. Smithsonian Institution, Washington, D.C. 1997.

VAN REGENMORTEL, M.H.V., FAUQUET, C.M., BISHOP, D.H. L., CARSTENS, E.B., ESTES, M.H., LEMON, S.M., MANILOFF, J., MAYO, M.A., MCGEOCH, D.J., PRINGLE, C.R. \& WICKNER, R.B. Virus Taxonomy: Seventh report of the International Committee on Taxonomy of Viruses. San Diego, CA. Academic Press. 2000.

WEBER, K. A. \& SEHGAL, O. P. Subgenomic RNAs in virions of southern bean mosaic virus. Phytopathology 72:909-913. 1982.

WEINTRAUB, M. \& RAGETLI, H.W.J. Electron microscopy of the bean and cowpea strains of Southern Bean Mosaic Virus within leaf cells. Journal of Ultrastructural Research 32:167-189. 1970. 